\title{
ESTUDOS SOBRE DIFFLUGIA LOBOSTOMA LEIDY (PROTISTA, RHIZOPODA, TESTACEALOBOSEA)
}

\author{
Vladimir Stolzenberg Torres ${ }^{1}$
}

\begin{abstract}
Studies about Difflugla lobostom 1 Leidy (Protista, Rhizopoda, TestaCealobosea). This paper reports feeding habitats, most commom test constitution, and growing curvature obtained under cultivation conditions, and the knowing distribution and most commom Difflugia lobostoma.

KEYWORDS. Difflugia lobostoma, cultivation, growing curvature, Protista.
\end{abstract}

Atualmente Difflugia lobostoma é certamente um dos testáceos mais conhecidos em todo o mundo (ProwazeK 1910; RUA 1912; PINTO 1925; GAUTIERLiĖVRE \& THOMAS 1958; THOMAS 1959; DiONI 1970; VuCETICH 1970, 1972, 1973a,b, 1976,1978; GREEN 1975; OGdEN \& HEDLEy 1980; CHARdEZ 1967, 1975, 1987, 1990; SABRI 1988; CHARDEZ et al. 1989; TORRES \& JEBRAM 1994), apresentando uma testa aglutinada de partículas inorgânicas sobre em substrato orgânico. GAUTIER-LiÈvRE \& THOMAS (1958) chegam a afirmar que este protista é cosmopolita.

Dos trabalhos anteriormente citados, a grande maioria é de caráter sistemático, como CHARDEZ (1987) que cita três variedades raras ocorrentes na Bélgica porém, os que apresentam uma outra conotação, como CHARDEZ et al. (1989), que apresenta um aspecto limnológico e SABRI (1988) que faz um estudo ecológico.

A importância desta espécie, do ponto de vista sistemático e especialmente ecológico, representa o objetivo e a justificativa para a realização de um estudo específico a respeito da mesma.

\section{MATERIAL E MÉTODOS}

O estudo de $D$. lobostoma foi estabelecido com a observação de amostras provenientes de habitats diversos bem como de diferentes regiões, a saber, os municípios de Porto Alegre, Minas do Butiá e Cambará do Sul (Rio Grande do Sul); Mundo Novo (Mato Grosso do Sul); além de exemplares mantidos em cultivo, provenientes de Torres (Rio Grande do Sul).

Os exemplares foram extraídos por espremedura de raízes de plantas flutuantes como Eichhornia spp. e Salvinia spp. - processo este, através do qual, toma-se a macrófita e espreme-se com a mão o conteúdo de suas raízes para o interior de um frasco; por pipetagem de amostras líquidas; coleta entre filóides de Briófitas; e isolamento para cultivo e clonagem conforme TORRES \& JEBRAM $(1993,1994)$.

1) Laboratório de Protistologia, Instituto de Biociências, Pontificia Universidade Católica do Rio Grande do Sul. Avenida Ipiranga 6681, prédio 12, 90619-900 Porto Alegre, Rio Grande do Sul, Brasil 


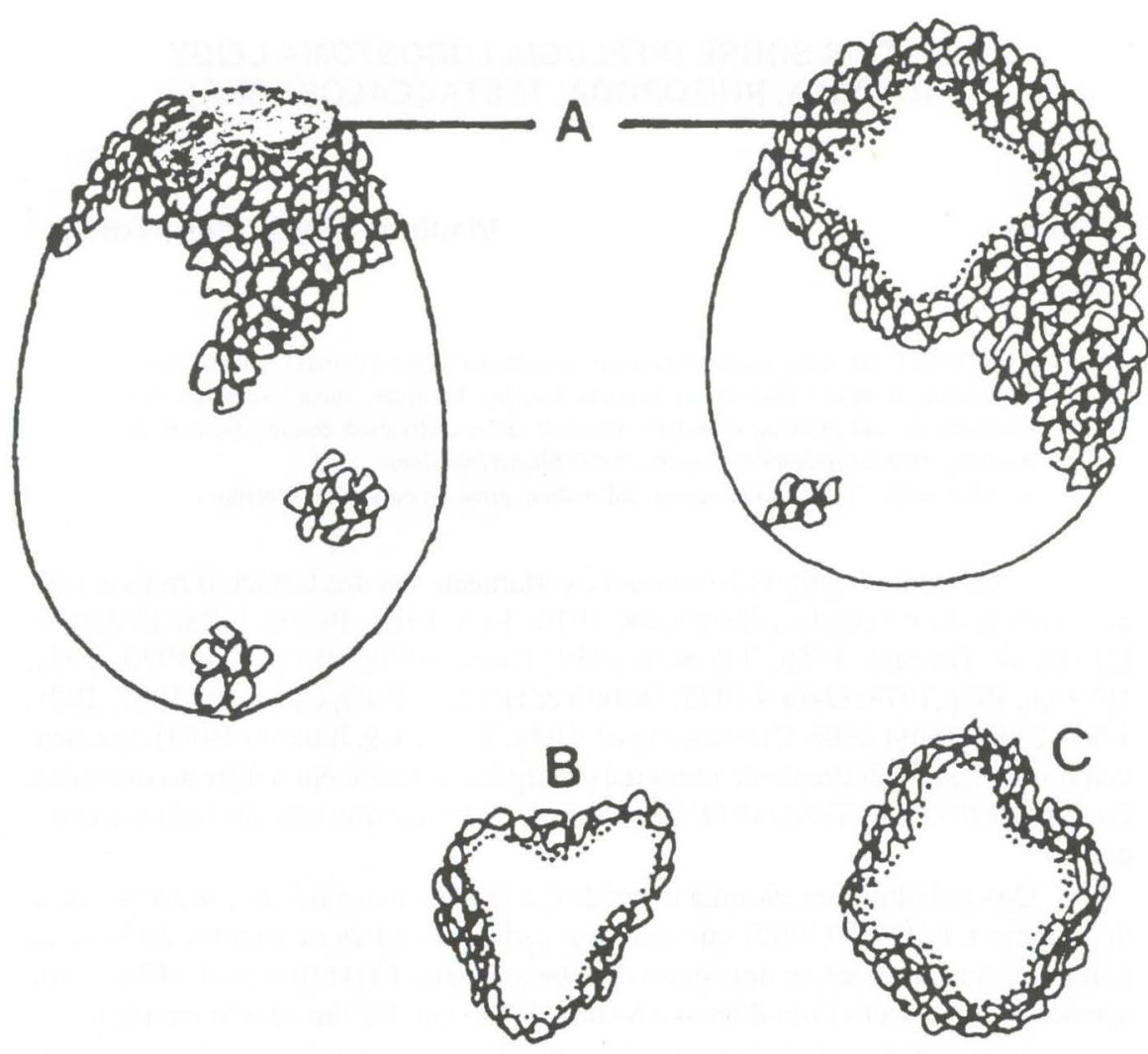

Fig. 1. Difflugia lobostoma lobostoma. (A) Visão da abertura pilomar; (B-C) algumas variações no números de lóbulos do piloma.

Os indivíduos postos em cultivo foram submetidos a diferentes tratamentos alimentares, conforme TORRES \& JEBRAM (1993), a fim de verificar o comportamento alimentar, sendo observado em paralelo o comportamento em amostras in natura. Neste caso considerando as amostras coletadas com sua composição original sem alterações introduzidas.

Aos indivíduos em cultivo foi fornecida sílica em pó e sílica espessante a fim de proporcionar material para a confecção da nova testa, por ocasião da reprodução.

Para o estudo da dinâmica de crescimento utilizou-se três clones obtidos a partir de um indivíduo coletado no Parque Estadual da Guarita (Torres, Rio Grande do Sul), com meio de cultivo "D/4A de Jebram", sílica em pó e espessante em quantidades à $100 \mathrm{mg} / \mathrm{dl}$ para a confecção das testas, e como alimento Chroomonas caudata Utermöhl, 1925, a qual já em outras oportunidades revelou-se uma ótima opção alimentar para protistas, conforme citam JEBRAM (1993) e TORRES \& JEBRAM (1993, 1994). 
Os clones utilizados não sofreram qualquer injúria aparente por ocasião de seu isolamento, pois o máximo cuidado foi tomado a fim de evitar que uma injúria ocorrente acidentalmente viesse a prejudicar o desenvolvimento do estudo da curva de crescimento dos clones.

As ocorrências conhecidas de D. lobostoma foram plotadas sobre um mapa mundial, projeção mista de Mercator e Moll Weide, a fim de verificar sua distribuição mundial e cosmopolitismo.

\section{RESULTADOS}

\section{1) Variedade e formas}

As variações morfológicas ocorrentes em D. lobostoma (Figs 1-5, Tab. I) levou à nomeação de uma série de variedades e mesmo de formas dentro das variedades, conforme GAUTHIER-LiÈvre \& THOMAS (1958), CHARDEZ (1967) e VUCETICH (1973a).

Tabela I. Variedades morfológicas de Difflugia lobostoma.

\begin{tabular}{|c|c|c|c|}
\hline Variedades e formas & Autores & Diâmetro $(\mu \mathrm{m})$ & Ocorrència conhecida \\
\hline var. Iobostoma Leidy & $\begin{array}{l}\text { Leidy } \\
\text { Gauthier-Lièvre \& Thomas } \\
\text { Vucetich } \\
\text { Chardez } \\
\text { Torres e Jebram }\end{array}$ & $\begin{array}{c}? \\
68-120 \\
65-110 \\
?-54-? \\
65-110\end{array}$ & $\begin{array}{l}\text { África } \\
\text { Brasil } \\
\text { Argentina } \\
\text { Chile } \\
\text { Brasil }\end{array}$ \\
\hline $\begin{array}{l}\text { var. lobostoma forma multilobata } \\
\text { Gauthier-Lièvre \& Thomas }\end{array}$ & $\begin{array}{l}\text { Vucetich } \\
\text { Gauthier-Lièvre \& Thomas } \\
\text { Chardez }\end{array}$ & $\begin{array}{l}90-120 \\
65-100 \\
?-46-?\end{array}$ & $\begin{array}{l}\text { Argentina } \\
\text { África } \\
-\end{array}$ \\
\hline var. truncata Playfair & Chardez & $?$ & África \\
\hline var. globulosa Playfair & $\begin{array}{l}\text { Gauthier-Lièvre \& Thomas } \\
\text { Playfair } \\
\text { Chardez }\end{array}$ & $\begin{array}{c}78-110 \\
? \\
?\end{array}$ & $\begin{array}{l}\text { África } \\
\text { Austrália } \\
\text { França, Chile }\end{array}$ \\
\hline $\begin{array}{l}\text { var. globulosa forma multilobata } \\
\text { Gauthier-Lièvre \& Thomas }\end{array}$ & $\begin{array}{l}\text { Gauthier-Lièvre \& Thomas } \\
\text { Chardez }\end{array}$ & $\begin{array}{c}80-110 \\
?\end{array}$ & Africa \\
\hline $\begin{array}{l}\text { var. tuberosa Gauthier-Lièvre \& } \\
\text { Thomas }\end{array}$ & $\begin{array}{l}\text { Gauthier-Lièvre \& Thomas } \\
\text { Chardez }\end{array}$ & $\begin{array}{c}70-95(110) \\
?\end{array}$ & África \\
\hline $\begin{array}{l}\text { var. tuberosa forma multilobata } \\
\text { Gauthier-Lièvre \& Thomas }\end{array}$ & $\begin{array}{l}\text { Gauthier-Lièvre \& Thomas } \\
\text { Chardez }\end{array}$ & $\begin{array}{c}125-130 \\
?\end{array}$ & África \\
\hline var. cornuta Gauthier-Lièvre \& Thomas & $\begin{array}{l}\text { Gauthier-Lièvre \& Thomas } \\
\text { Chardez }\end{array}$ & $\begin{array}{c}72-90 \\
?\end{array}$ & África \\
\hline
\end{tabular}

\section{2) Descrição}

Segundo TORRES \& JeBram (1994) Difflugia lobostoma apresenta-se sem gargalo e nem colarete; testa formada por partículas areanáceas, no caso do cultivo formada por partículas de sílica em pó e sílica espessante, com granulação próxima ou inferior a 1-2 $\mu \mathrm{m}$, irregular, com interstícios fechados por pedrinhas de mais fina granulação; presença eventual de frústulas de diatomáceas (Fig. 5). O piloma pode variar de tri a hexalobado, sendo esta última condição a mais frequente. Lóbulos profundos, de bordos retos e quase paralelos, visíveis como uma edentação na vista lateral da testa. 


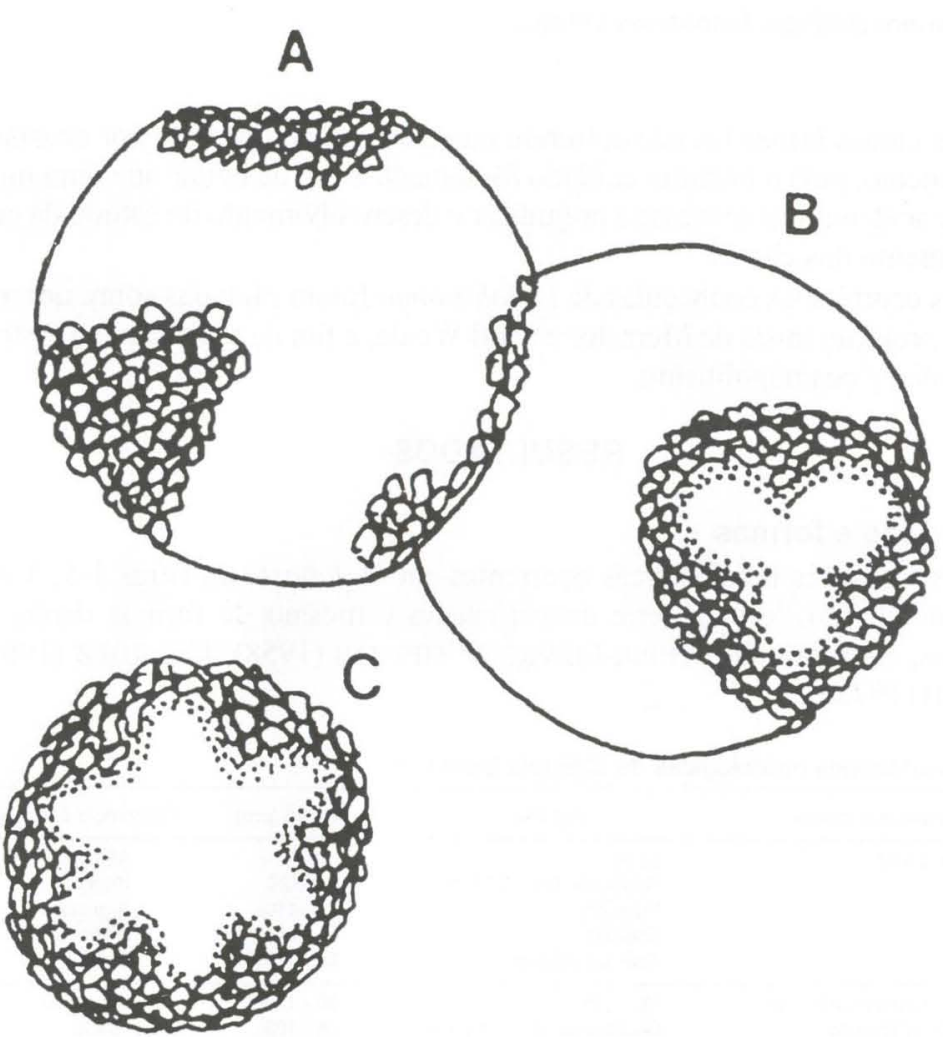

Fig. 2. Difflugia lobostoma globulus Playfair, 1917. (A-B) Vistas lateral e oral da testa; (C) visão da abertura pilomar.

Os indivíduos cultivados apresentaram como dimensões: diâmetro apical $65,0-110,0 \mu \mathrm{m}$; altura $85,0-120,0 \mu \mathrm{m}$; diâmetro do piloma $30,0-40,0 \mu \mathrm{m}$; relação altura/diâmetro $0,78-1,85 \mu \mathrm{m}$.

Observou-se que em condições naturais D. lobostoma pode utilizar aparentemente qualquer material disponível para a confecção de sua testa, indo de grãos de areia a frústulas de diatomáceas (STOUT \& WALKER 1976) assim, em exemplares provenientes do município de Minas do Butiá constatou-se a presença de partículas de carvão mineral na testa (Fig. 6).

Em cultivo verificou-se a eventual utilização de frústulas de diatomáceas com um índice de aproximadamente 5,0-9,5\%, mesmo quando da ampla disponibilidade de partículas de sílica.

STOUT \& WALKER (1976) apontam a presença de diferentes elementos químicos na testa de Difflugia e outros gêneros, sendo como constituintes principais sílicio e alumínio e ocorrendo traços de sódio e cloro (Tab. II).

\section{3) Ambientes}

Difflugia lobostoma foi observada em dois ambientes, a saber: dulceaqüícola e muscícola. 


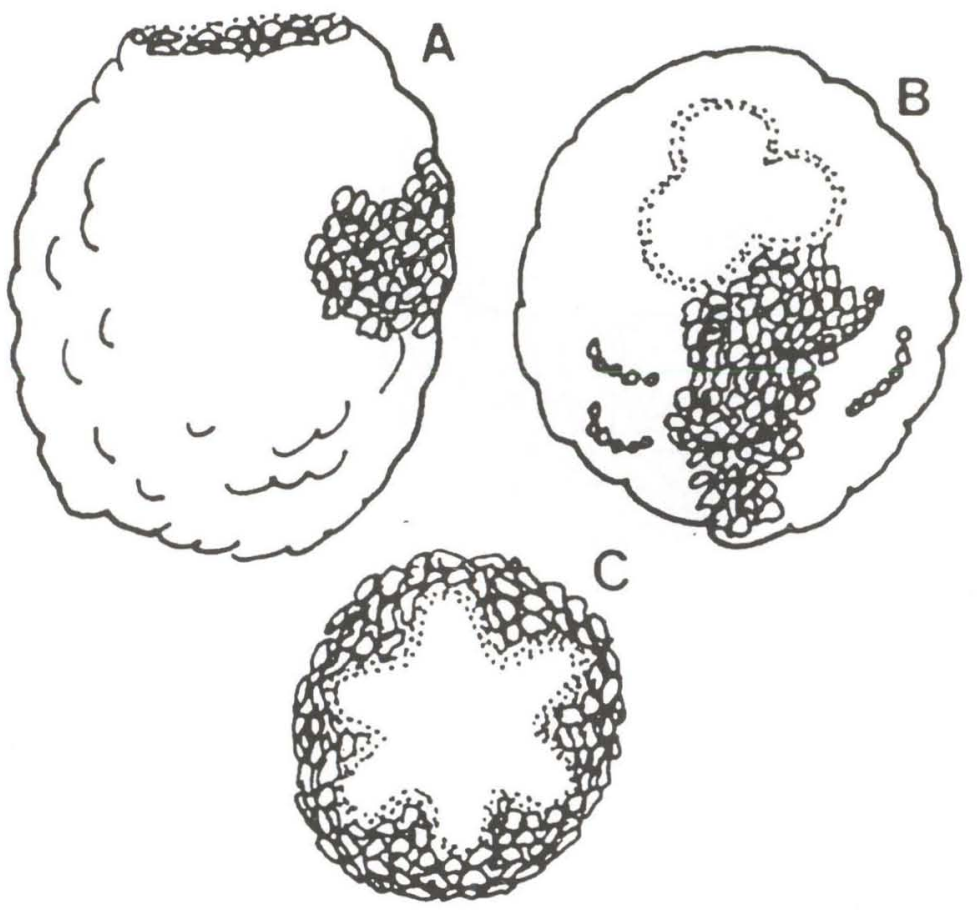

Fig. 3. Difflugia lobostoma tuberosa Gauthier-Lièvre \& Thomas, 1958. (A-B) Vistas lateral e oral da testa; (C) visão da abertura pilomar.
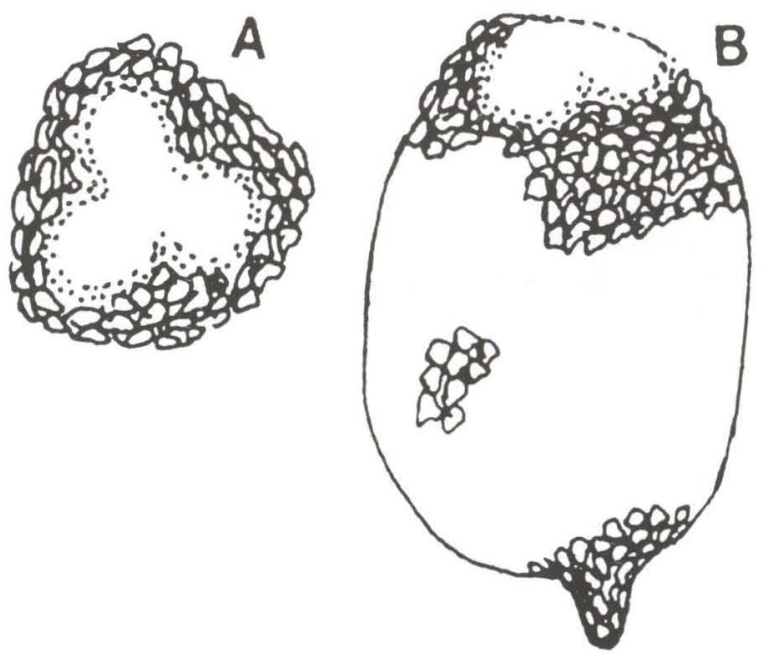

Fig. 4. Difflugia lobostoma cornuta Gauthier-Lièvre \& Thomas, 1958. (A) Vista da abertura pilomar; (B) vista lateral da testa. 


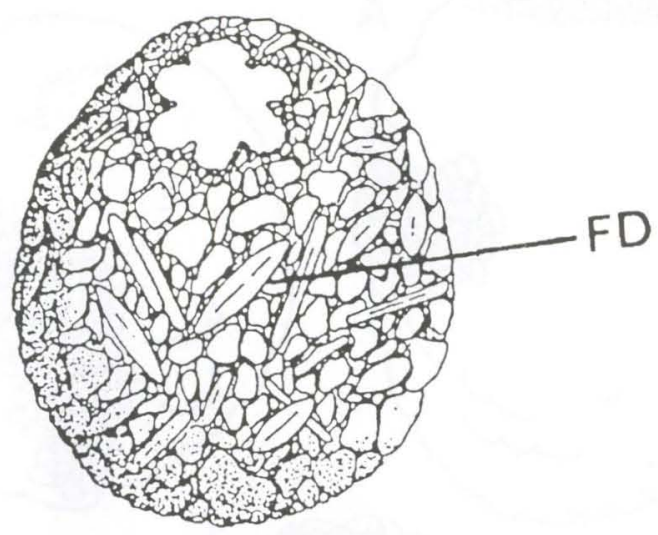

Fig. 5. Testa de Difflugia lobostoma multilobata Gauthier-Lièvre \& Thomas, 1958 com presença de frústulas de diatomáceas (FD) em sua composição. (modificado de VucCETICH 1973).

Tabela II. Constituintes das testas de alguns gêneros de testáceos (STOUT \& WALKER 1976).

\begin{tabular}{cccc}
\hline Material examinado & Constituinte principal & Outros constituintes significativos & Traços de constituintes \\
\hline Difflugia & $\mathrm{Si}, \mathrm{Al}$ & - & $\mathrm{Na}, \mathrm{Cl}$ \\
Centropyxis & $\mathrm{Si}$ & $\mathrm{Al}, \mathrm{K}, \mathrm{Ca}$ & - \\
Cyclopyxis & $\mathrm{Si}$ & $\mathrm{Al}, \mathrm{K}$ & $\mathrm{Ca}$ \\
Euglypha & $\mathrm{Si}$ & - & - \\
\hline
\end{tabular}

O ambiente dulceaqüícola caracterizou-se por ecossistemas lóticos e lênticos, utilizando a classificação de ODUM (1988). Em geral o protista foi observado associado a um substrato; raramente foi constatada sua presença em estado planctonte.

No ambiente muscícola encontramos duas situações: a área de turfeiras e a das briófitas tipicamente terrestres. Em ambas observou-se exemplares vivos deste protista, sendo que THOMAS (1959) relata a presença desta espécie em briófitas tipicamente terrestres, mas afirma que a mesma apresenta a incontestável afinidade aquática.

\section{4) Hábitos Alimentares}

Dentre os hábitos alimentares verificou-se em cultivo a utilização de uma ampla gama de microalgas como Cosmarium sp., Closterium sp., Euglena sp., Micrasterias sp., Pediastrum duplex Meyen, 1892, Spirotaenia sp., Coelastrum sp., Cryptomonas sp., de diversas diatomáceas penais, de ciliados como Paramaecium sp., e Colpidium sp., e mesmo testáceos de outros taxa. Nas amostras coletadas observou-se a fagocitose de microcrustáceos (Cocepoda e Ostracoda), bem como de pequenos ácaros (Figs 7-9). A capacidade de crescimento é um índice único, este inerente a uma população. 

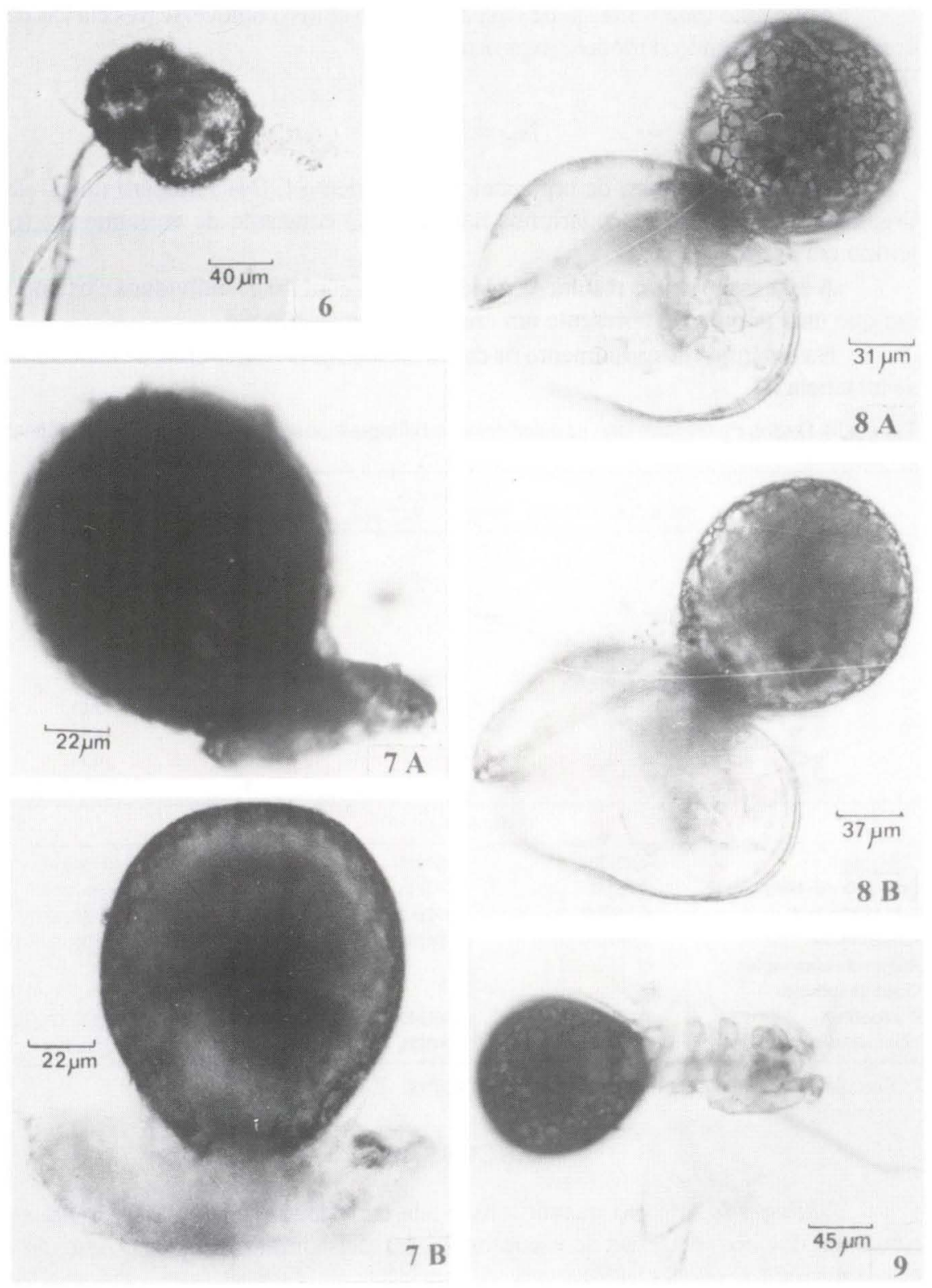

Figs 6-9. (6) Testa apresentando partículas de carvão; (7) fagocitose de microácaros, (A) foco no microácaro; (B) foco em Difflugia lobostoma; (8A-B) Ostracoda sendo fagocitado; (9) Difflugia lobostoma fagocitando um Copepoda. 
Aplicando uma Equação de Crescimento ao cultivo obteve-se três curvas de crescimento, segundo o modelo exponencial:

$$
\mathrm{N}_{\mathrm{t}}=\mathrm{N}_{0} \cdot e^{\mathrm{K} \cdot \mathrm{t}}
$$

Onde: $\left(\mathrm{N}_{\mathrm{t}}\right)$ número de organismos no momento $\mathrm{t} ;\left(\mathrm{N}_{0}\right)$ número inicial de organismos; $(e)$ base de logaritmos naturais; $(\mathrm{K})$ constante de crescimento; $(\mathrm{t})$ tempo em horas.

A expressão acima resulta da relação entre o número de indivíduos e o tempo em que uma população apresente um crescimento exponencial.

Na dinâmica de crescimento os dados obtidos para os três clones encontramse na tabela III.

Tabela III. Dados de crescimento populacional de Difflugia lobostoma em condições de cultivo laboratorial.

\begin{tabular}{|c|c|c|c|c|}
\hline $\begin{array}{c}\text { Tempo } \\
\text { (h) }\end{array}$ & $\begin{array}{c}\text { Clone } 1 \\
\text { Número de individuos }\end{array}$ & $\begin{array}{l}\text { Clone } 1 \\
\text { Número de indivíduos }\end{array}$ & $\begin{array}{c}\text { Clone } 1 \\
\text { Número de individuos }\end{array}$ & $\begin{array}{l}\text { Média dos } \\
\text { Clones }\end{array}$ \\
\hline 0,00 & 1 & 1 & 1 & 1 \\
\hline 23,67 & 1 & 1 & 1 & 1 \\
\hline 48,04 & 1 & 1 & 1 & 1 \\
\hline 71,06 & 2 & 1 & 2 & 1,67 \\
\hline 95,04 & 2 & 2 & 2 & 2 \\
\hline 122,44 & 2 & 2 & 2 & 2 \\
\hline 149,01 & 2 & 2 & 3 & 2,33 \\
\hline 172,05 & 2 & 3 & 3 & 2,67 \\
\hline 195,52 & 3 & 4 & 6 & 4,33 \\
\hline 220,15 & 4 & 4 & 8 & 5,33 \\
\hline \multicolumn{5}{|c|}{ Dados estatisticos * } \\
\hline Constante & $-0,039336$ & $-0,207377$ & $-0,207721$ & 0,154010 \\
\hline Constante exponencializada & 0,961428 & 0,812713 & 0,812434 & 0,857264 \\
\hline Erro padrăo de Y & 0,198202 & 0,183663 & 0,222968 & 0,156333 \\
\hline Raiz quadrada & 0,842076 & 0,908552 & 0,916287 & 0,937686 \\
\hline Número de observações & 10 & 10 & 10 & 10 \\
\hline Graus de liberdade & 8 & 8 & 8 & 8 \\
\hline Coeficiente $\mathrm{X}$ & 0,005783 & 0,007315 & 0,009321 & 0,007663 \\
\hline Erro padrăo do coeficiente & 0,000885 & 0,000821 & 0,000996 & 0,000698 \\
\hline
\end{tabular}

*. Calculados a partir dos respectivos valores acima.

\section{DISCUSSÃO}

Difflugia lobostoma quando observada em estado planctonte apresenta-se flutuando devido à presença de vácuolos de gás, estes similares aos de D. mitriformis, conforme TORRES (1996).

A fagocitose de invertebrados por protistas não é uma novidade. ANDERSON \& BÉ (1976) relatam a fagotrofia praticada pelo foraminífero Hastigerina pelagica que foi observado capturando e utilizando como alimento náuplios de Artemia sp. e copépodes. SWANBERG \& ANDERSON (1985) citam que o radiolário Spumellaria sp. (Radiolaria, Ralycystinea, Spumellarida) utilizou, como alimento, copépodes, 
formas jovens de moluscos e ostracodes. De acordo com ANDERSON et al. (1979), algumas espécies de foraminíferos são onívoros, alimentando-se de algas e tecidos animais, como tecido adiposo, muscular, epitelial, nervoso e mesmo outros tecidos. É a primeira ocorrência desta natureza que se conhece para o grupo de Testacealobosea.

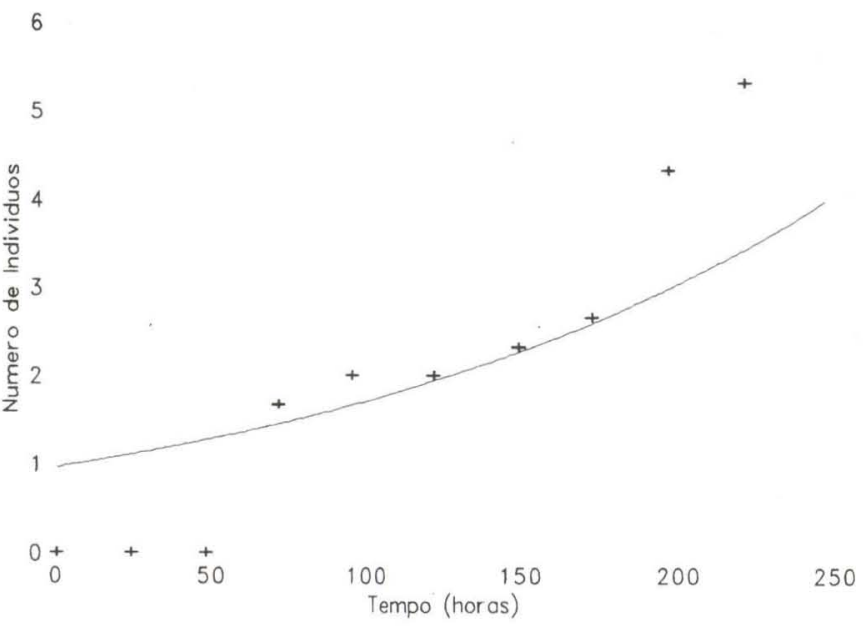

Fig. 10. Curva de crescimento obtida para o clone 1.

6

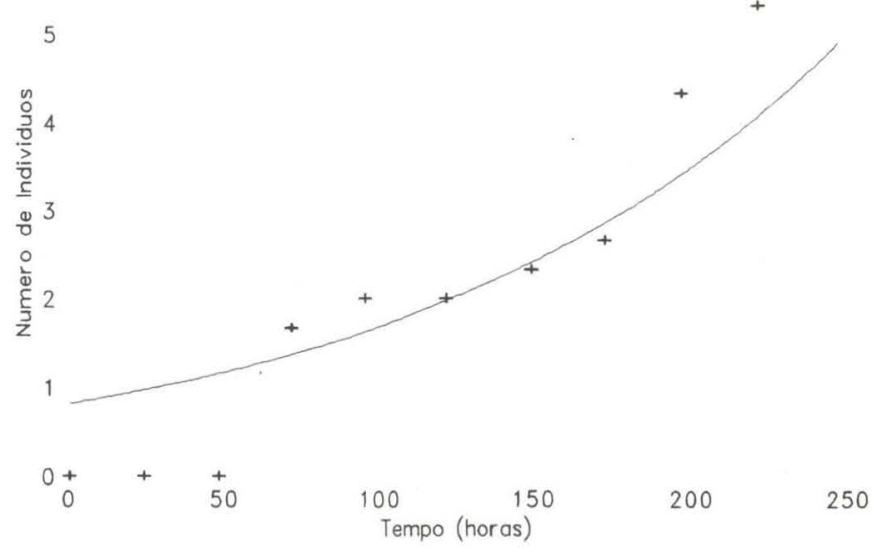

Fig. 11. Curva de crescimento obtida para o clone 2 .

De acordo com LEVEAU \& BOUTX (1985) quando um organismo é introduzido num meio de cultivo que lhe é adequado, ele desenvolve aí uma atividade relacionada com sua composição e com as condições que o rodeiam, isto é, o 
organismo se reproduz. O crescimento microbiano pode ser considerado como um conjunto de reações químicas em cadeia, que levam à síntese dos constituintes da aumentada biomassa microbiana obtida no final do processo.

Embora GAUTHIER-LIĖVRE \& THOMAS (1958) possam considerar D. lobostoma cosmopolita, esta espécie não pode ser ainda, considerada como tal, o que pode ser facilmente comprovado através da figura 14 , onde se verifica a sua não ocorrência na Antártida, Ásia, Oceania, Américas do Norte e Central e Noroeste da América do Sul.

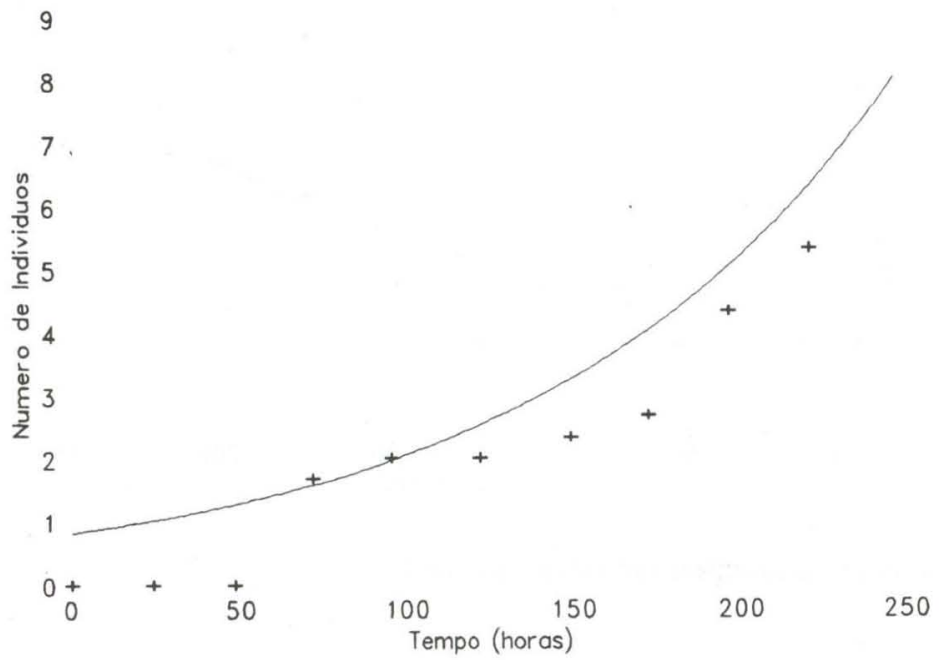

Fig. 12. Curva de crescimento obtida para o clone 3.

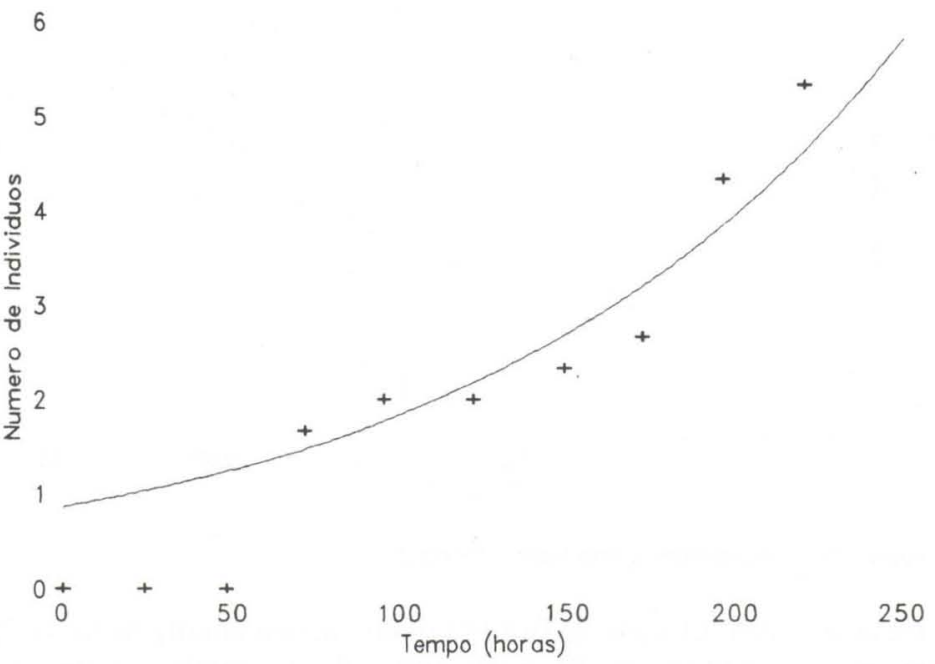

Fig. 13. Curva de crescimento média obtida para os clones 1, 2, 3 . 


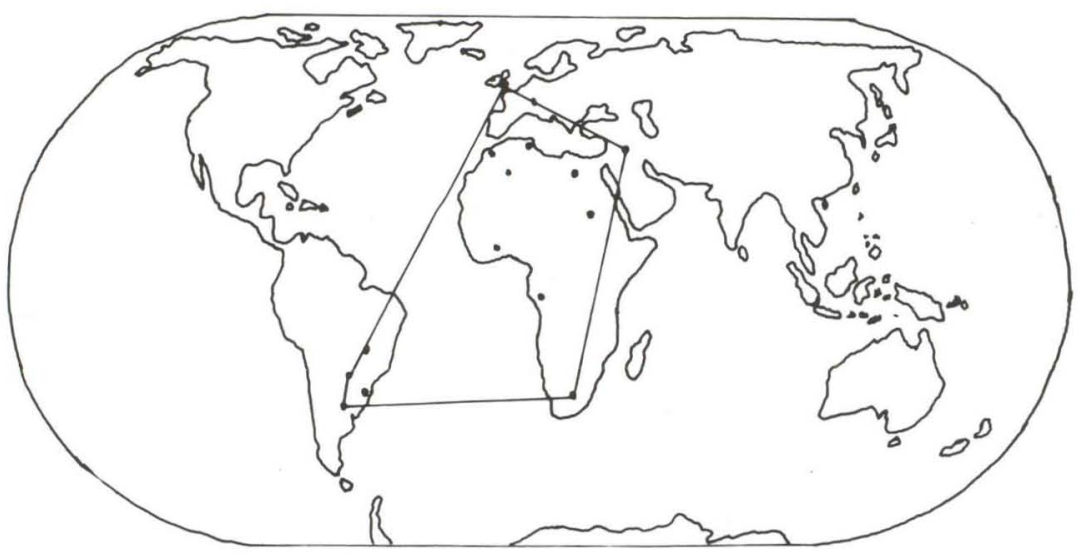

Fig. 14. Mapa mundi mostrando todos os locais onde se tem registro de ocorrência de Difflugia Iobostoma. Os pontos assinalam os locais de registro efetivo e a linha traçada marca o maior perímetro para uma ocorrência a partir dos dados disponiveis.

\section{CONCLUSÕES}

Difflugia lobostoma apresenta grande variedade de formas, podendo ocorrer formas ainda não detectadas, porém não se pode afirmar com toda a certeza que sejam efetivamente variações estáveis, ou que não sejam mesmo outras espécies.

Para isso faz-se necessário a realização de cultivos controlados e maiores estudos morfo-citométricos de cada sub-grupo. A testa pode, contudo, assumir variações morfológicas em função do material inorgânico utilizado para sua confecção, este bastante variável conforme o habitat do indivíduo.

Considerando o amplo espectro alimentar apresentado por este protista, constata-se a sua ampla importância como predador das teias alimentares microbiológicas, onde assume o papel de consumidor de diferentes níveis tróficos, relacionando-se assim diretamente o alimento, o qual pode ser desde microalgas até mesmo microcrustáceos.

Em geral, as curvas de crescimento dos clones mostraram características muito semelhantes. Considerando a curva média e como todas apresentam um desenvolvimento logarítmico a base " $e$ ", o que se encontra é uma curva de crescimento normal para os indivíduos testados, inferindo-se então que o meio aplicado - D/4 A (JeBram 1993), o silício e o alimento oferecido - Chroomonas caudata, são suficientemente adequados para um cultivo laboratorial desta espécie, bem como, a sua manutenção a nível de coleção.

AGRADECIMENTOS. Ao Laboratório Anakol pelo fornecimento da sílica espessante que possibilitou o cultivo de Difflugia lobostoma; e ao Prof. Dr. Luis Glock por todo apoio prestado, sendo este artigo resultante do Trabalho de Conclusão da Disciplina Dinâmica Populacional. 


\section{REFERÊNCIAS BIBLIOGRÁFICAS}

ANDERSON, O.R. \& A.W.H. BÉ. 1976. Cytochemical fine structure study of phagotrophy in a planctonic foraminifer, Hastigerina pelagica (d'Orbigny). Biol. Bull. 151 (3): 437-449.

ANDERSON, O.R.; N.R. SWANBERG \& P. BENNETT. 1979. Trophic activity of planktonic foraminifera. J. mar. biol. Ass. U.K. 59: 791-799.

Chardez, D. 1967. Histoire Naturelle des Protozoaires Thécamoebiens. Nat. Belges.: 1-100.

- 1975. Quelques Thecamoebiens de la Region de Butare (Zaire). Rev. Verviét. d'Hist. nat. 5: 7-9.

1987. Catalogue des Thecamoebiens de Belgique (Protozoa Rhizopoda Testacea). Not. Faun. Gembloux. 13: 1-19.

1990. Contribution a la conaissance des Thecamoebiens aquatiques du

Tyrol Allemand (Rhizopoda, Testacea). Acta Protozoologica 29 (2): 153-156.

Chardez, D.; L. BougarT \& CH. GaSPAR. 1989. Les Thecamoebiens du sapropele des etangs du Domaine des Epioux (Protozoa Rhizopoda Testacea). Biol. Jb. Dodonaea 57: 51-61.

DiONI, W. 1970. Taxocenos de Tecamebianos em Cuencas Islenas del Parana Medio, I. Acta Zool. Lill. 27: 201-238.

Gauthier-Lièvre, L. \& R. Thomas. 1958. Les genres Difflugia, Pentagonia, Maghrebia et Hooreradia (Rhizopodes Testacés) en Afrique. Arch. Protistenk. 103: $241-270$.

GReEN, J. 1975. Freewater Ecology in the Mato Grosso, Central Brazil, IV: Associations of Testate Rhizopoda. J. Nat. Hist. 9: 545-560.

Jebram, D.H.A. 1973. Métodos Básicos e Novos para o Cultivo de Protistas Livres. Comum. Mus. Ciênc. PUCRS 50: 3-20.

LeVeau, J.Y. \& M. BouIX . 1985. Cinéticas Microbianas, p.103-123. In: R. Scriban; A. Arnaud; C. KubiaK; C. Berset; H. Richard; J. Pourquie; J. GoURSAD; J. LARRIEU; J.P. VANDECASTEELE; J.Y. LEVEAU; J. DE ROSNAY; J. BocQueT; J.P. GuIraud; M. BouIX; M. BERnard; P. Galzy \& T. Dubuis (Eds). Biotecnologia. São Paulo, Manole, 489p.

Ogden, C.G. \& R.H. Hedley. 1980. An Atlas of Freswater Testate Amoebae. London, British Museum (Natural History), 222p.

ODUM, E.P. 1988. Ecologia. Rio de Janeiro, Editora Guanabara, 434p.

PINTO, C. 1925. Protozoários observados no Brasil. Mem. Inst. Oswaldo Cruz 18 (1): 211-302.

ProwAZEK, S. vON. 1910. Contribuição ao Conhecimento da Fauna de Protozoários do Brasil. Mem. Inst. Osw. Cruz, Rio de Janeiro, 2 (2): 149-158.

RUA, J.M. DE LA. 1912. Algunos Protozoos Tecamebianos de la Argentina. Physis: 43-46.

SABRI, A.W. 1988. Seasonal Variation of Testaceous Amoebae (Sarcodina, Protozoa) Population in the River Tigris. Arab. Gul J. Scient. Res. Agric. Biol. Sci. B6 (3): 439-448. 
Stout, J.D. \& G.D. WALKER. 1976. Discrimination of mineral particles in test formation by Thecamoebae. Trans. Amer. Micros. Soc. 95 (3): 486-489.

SWANBERG, N.R. \& O.R. ANDERSON. 1985. The nutrition of Radiolarians: Trophic activity of some solitary Spumellaria. Limnol. oceanogr. 30 (3): 646-652.

THOMAS, R. 1959. Les Thecamoebiens muscicoles et terricoles: notions d'écologie générale et comparative. Soc. Linn. Bordeaux 98: 27-53.

TORRES, V.S. 1996. Vácuolos de Gás e Flutuação em Difflugia mitriformis Wallich, 1864 (Protista, Rhizopoda, Testacealobosea). Revta bras. Zool. 13 (1):67-75.

TorreS, V.S. \& D.H.A. JeBram. 1993. Arcella gibbosa microsoma var.n. (Protozoa, Sarcodina, Testacea) descrição e observações feitas em seu cultivo. Biotemas 6 (2): 20-29.

1994. Amebas Testáceas Ocorentes na Região Metropolitana de Porto Alegre, RS. Biotemas 7 (1/2): 65-78.

VucceTich, M.C. 1970. Algunos Tecamebianos de la Provincia de Formosa. Neotropica 16 (49): 42-48.

1972. Tecamebianos del Eupleston de cuerpos da agua de la Provincia de Buenos Aires. Acta Zool. Lill. 29: 271-284.

.1973a. Estudio de Tecamebianos Argentinos en especial los del Domino Pampasico. Rev. Mus. La Plata. Zoologia 11 (108): 287-332.

1973b. Contibuicion al conocimiento de la Ecologia y Zoogeografia de los Tecamebianos Argentinos. Rev. Mus. La Plata. Zoologia 11 (109): 333 358.

1976. Tecamebianos del lago San Roque y de un ambiente lentico artificial vinculado al mismo (Cordoba, Argentina). Limnobios 1 (2): 29-34.

1978. Nuevos aportes al conocimiento de los Tecamebianos del Domínio Subtropical. Neotropica 24 (72): 79-90.

Recebido em 25.V.1995; aceito em 14.X.1996. 